\title{
Die schweizerische Unterstützung von Distriktsgesundheitssystemen in Entwicklungsländern
}

Nicolaus Lorenz, Eric Burnier et Marcel Tanner

\section{CpenEdition}

Journals

Édition électronique

URL : http://journals.openedition.org/sjep/1417

DOI : $10.4000 /$ sjep. 1417

ISSN : 1663-9677

Éditeur

Institut de hautes études internationales et du développement

Édition imprimée

Date de publication : 1 janvier 1994

Pagination : 185-205

ISSN : 1660-5926

\section{Référence électronique}

Nicolaus Lorenz, Eric Burnier et Marcel Tanner, « Die schweizerische Unterstützung von Distriktsgesundheitssystemen in Entwicklungsländern », Schweizerisches Jahrbuch für 


\title{
Die schweizerische Unterstützung von Distriktsgesundheitssystemen in Entwicklungsländern
}

\author{
Nicolaus Lorenz, Eric Burnier und Marcel Tanner
}

“Der Distrikt ist der natürliche Begegnungspunkt, wo sich die basisnahe Planung und Organisation mit der zentralen Planung und Unterstützung treffen und ist somit der Ort an dem Gemeindebedürfnisse und nationale Prioritäten sich vereinigen können."

Tarimo(1), 1991

Nach der chronologischen Darstellung der Entwicklung der schweizerischen Entwicklungszusammenarbeit folgt nun die Darstellung einiger ausgewählter Beispiele gesundheitsspezifischer Unterstützungsmassnahmen, die in den letzten Jahren von der Schweiz ausgingen.

Trotz aller Unterschiede, auf die im einzelnen einzugehen sein wird, haben die dargestellten Projekte eines gemeinsam: Sie sind in der Peripherie, in einem Gesundheitsdistrikt, angesiedelt.

Die Weltgesundheitsorganisation (WHO (2), 1988) spricht von Gesundheitsdistriktssystemen, die sämtliche Aspekte von Gesundheit bzw. Behandlung von Krankheit umfassen. Dazu gehört die Selbstbehandlung und Krankheitsvorsorge, wie sie in der Familie stattfindet. Dann aber auch die staatlichen oder nichtstaatlichen Einrichtungen, die sowohl kurative als auch präventive Dienstleistungen anbieten. Vor allem im Bereich der Krankheitsbehandlung ist die Mehrstufigkeit der Versorgung, je nach Bedarf und Schwere des Krankheitsbildes hervorzuheben. 
Schematisch lässt sich dies mit einer Gesundheitspyramide vergleichen. In einem idealtypischen Referenzsystem wird eine erkrankte Person zunächst in der Familie, und wenn sich dort das Problem nicht lösen lässt, meistens in einer ersten Versorgungsstufe, bspw. einem einfachen Dispensarium behandelt. Wenn die dort zur Verfügung stehenden Behandlungsmöglichkeiten nicht ausreichen sollten, erfolgt die Überweisung in das Distriktsspital, und dann über verschiedene Versorgungsstufen bis hin zu einem Universitätskrankenhaus. Die meisten Gesundheitsprobleme lassen sich aber innerhalb eines Distriktsgesundheitssystems behandeln.

Das Distriktskonzept stellt eine konsequente Weiterentwicklung des Basisgesundheitsgedankens dar (Medicus Mundi International (3), 1989), indem es dem in der Basisgesundheit enthaltenen gesamthaften Ansatz der Gesundheitsversorgung und -erhaltung eine räumliche und sozio-ökologisch verhältnismässig homogene Dimension verleiht. Die Definition eines Distriktes hängt sehr stark von den örtlichen geographischen und administrativen Gegebenheiten ab. Zwischen 50'000 und 500'000 Menschen leben in einem Distrikt. Eine erste Versorgungsstufe besteht beispielsweise aus einigen Dispensarien und Ambulatorien, die je nach Kontext ein unterschiedlich grosses Paket an Gesundheitsdienstleistungen (ambulante Grundversorgung) anbieten. Beim Personal handelt es sich in vielen Ländern um medizinische Assistenzkräfte, manchmal - wie bspw. in der Schweiz - erfüllt aber auch medizinisches Personal diese Aufgabe. Über der ersten Versorgungsstufe findet sich ein übergeordnetes Gesundheitszentrum, das die Referenzfunktion für die Dispensarien übernimmt, sowie zusätzliche Dienstleistungen, wie chirurgische Eingriffe oder technisch aufwendigere Laborleistungen, anbietet.

Meistens findet sich auch qualifizierteres Personal, bspw. Ärzte auf dieser Versorgungsstufe. Oft, aber nicht immer, entspricht die Aufteilung der Einzugsgebiete für die Gesundheitsdienste allgemein administrativen, staatlichen Strukturen, die für das Ressourcenmanagement, die Planung und deren Umsetzung verantwortlich sind. Die Gesundheitsdienstleistungen können sowohl von staatlichen als auch von privaten Einrichtungen erbracht werden.

Wenn man die qualitativen Aspekten des Personals ausklammert, entsprechen auch in der Schweiz viele kantonale Gesundheitsversorgungssysteme durch die niedergelassenen Allgemeinärzte und das übergeordnete Spital (und die niedergelassenen Fachärzte) der oben aufgeführten Definition eines Distriktsgesundheitssystems.

Der Anteil der distriktsorientierten Unterstützung an der gesamten schweizerischen Entwicklungszusammenarbeit ist im einzelnen schwer abzuschätzen. Auf Grund von Angaben der Direktion für Entwicklungszusammenarbeit und humanitäre Hilfe (DEH) und der schon erwähnten Befragung von nichtstaatlichen im Gesundheitsbereich tätigen Organisationen kann man davon ausgehen, dass von 1983 bis 1992 etwa ein Drittel der staatlichen und ein grosser Teil der nichtstaatlichen medizinischen Entwicklungszusammenarbeit zur Stützung von Distriktsgesundheitssystemen eingesetzt worden ist. Es ist festzuhalten, dass in den vergangenen beiden Jahren im Bereich der Unterstützung der DEH eine deutliche 
Verschiebung zu Gunsten von distriktsorientierten Massnahmen stattgefunden hat und diese seit 1990 fast $60 \%$ der Entwicklungszusammenarbeit im Gesundheitssektor ausmachen.

Der geographische Schwerpunkt dieser Aktivitäten der DEH liegt vor allem in Afrika südlich der Sahara. Nichtstaatliche Organisationen und Einrichtungen haben ebenfalls Afrika als Schwerpunktregion, aber es finden sich auch wichtige Aktivitäten in Asien und Lateinamerika.

Die schweizerischen Aktivitäten auf Distriktsebene können zur Vereinfachung der Darstellung in fünf Kategorien eingeteilt werden:
A. Basisnahe Aktivitäten
B. Krankenhausaktivitäten
C. Integrierte Distriktsunterstützungen
D. Distriktsgesundheitssystemforschung
E. Regionale, nationale und internationale Massnahmen, die das Distriktskonzept unterstützen

Im folgenden sollen nun ausgewählte Beispiele der schweizerischen Entwicklungszusammenarbeit dargestellt werden. Es handelt sich um eine Auswahl, die keinen Anspruch auf Vollständigkeit erhebt. Ausserdem haben viele der dargestellten Projekte unterschiedliche Aspekte, die sich nicht in das oben vorgegebene Schema einfügen lassen. Bei der Zuteilung war der Schwerpunkt der dargestellten Interventionen ausschlaggebend. Die Beispiele zeigen exemplarisch die wesentlichen Inhalte und die Schwierigkeiten der schweizerischen Entwicklungszusammenarbeit im Gesundheitssektor. Die nachfolgende Tabelle gibt eine vereinfachende Übersicht über die wesentlichen Charakteristika der verschiedenen Ansätze.

\section{A) Basisnahe Aktivitäten}

Die Nichtregierungsorganisation "Sustaining Health Assistance for Life Ordaining Ministry" (Shalom) in Oroquieta City, Philippinen unterstützt mit Mitteln des Hilfswerks Evangelischer Kirchen der Schweiz (HEKS) 265 Familien. Die Familienoberhäupter sind Fischer, Kleinbauern und Arbeiter. Das Projekt umfasst gesundheitsrelevante Aktivitäten, wie Gesundheitsbewusstseinsseminare, ein Gesundheitstraining und ein Familienplanungsprogramm. Darüberhinaus sind aber auch einkommensfördernde Massnahmen, wie Seifenherstellung und Kleintiervermarktung, Bestandteil dieses Projektes. Diese sollen die sozio-ökonomische Basis der Zielbevölkerung verbessern.

In Tomohon/Nord-Sulawesi unterstützt die schweizerische Ostasienmission vor allem präventive Aktivitäten der Evangelischen Minahasakirche. Im Bereich der Versorgung von Schwangeren und Kleinkindern ist beispielsweise kaum ausgebildetes Gesundheitspersonal und medizinische Infrastruktur vorhanden. Dies führt dazu, dass die überwiegende Zahl der Frauen bei sich zu Hause gebären 


\begin{tabular}{|c|c|c|c|c|c|}
\hline & Zielgruppe & $\begin{array}{l}\text { Interventions- } \\
\text { ebene }\end{array}$ & Methoden & $\begin{array}{l}\text { Beteiligte Institutionen } \\
\text { und Organisationen }\end{array}$ & $\begin{array}{l}\text { Finanzierungsquellen } \\
\text { und Finanzmittelbedarf }\end{array}$ \\
\hline Basisansatz & $\begin{array}{l}\text { marginalisierte } \\
\text { Bevölkerungsgrup- } \\
\text { pen, benachteiligte } \\
\text { Gruppen, Frauen, } \\
\text { Kinder klar um- } \\
\text { schriebene Bevöl- } \\
\text { kerungsgruppen } \\
\end{array}$ & $\begin{array}{l}\text { Familie, } \\
\text { Kleingruppe }\end{array}$ & $\begin{array}{l}\text { Finanzielle, selten person- } \\
\text { nelle Unterstützung, } \\
\text { Unterstützung lokaler } \\
\text { Initiativen }\end{array}$ & $\begin{array}{l}\text { Schweizerische NROs } \\
\text { und NROs aus Partner- } \\
\text { ländern }\end{array}$ & $\begin{array}{l}\text { Vor allem nichtstaatliche } \\
\text { Partner in der Schweiz } \\
\text { und Partnerländer: } \\
\text { relativ geringer Finanz- } \\
\text { mittelbedarf }\end{array}$ \\
\hline Spitalansatz & $\begin{array}{l}\text { Patienten und } \\
\text { potentielle } \\
\text { Nutzer/innen } \\
\text { von Gesund- } \\
\text { heitseinrichtungen }\end{array}$ & $\begin{array}{l}\text { Gesundheits- } \\
\text { strukturen, oft nur } \\
\text { Krankenhaus }\end{array}$ & $\begin{array}{l}\text { Finanzielle, materielle und } \\
\text { tlw. personnelle Unter- } \\
\text { stützung }\end{array}$ & $\begin{array}{l}\text { Schweizerische NROs } \\
\text { und NROs aus } \\
\text { Partnerländern }\end{array}$ & $\begin{array}{l}\text { Nichtstaatliche und } \\
\text { staatliche Organisa- } \\
\text { tionen in der Schweiz } \\
\text { und Partnerländer, } \\
\text { mittlerer bis hoher } \\
\text { Finanzmittelbedarf }\end{array}$ \\
\hline $\begin{array}{l}\text { Gesamthafter } \\
\text { Distriktansatz }\end{array}$ & $\begin{array}{l}\text { Allgemeine } \\
\text { Bevölkerung, } \\
\text { die in einem } \\
\text { definierten Ein- } \\
\text { zugsgebiet lebt }\end{array}$ & $\begin{array}{l}\text { Gesundheits- } \\
\text { strukturen, und } \\
\text { Distrikt, Klein- } \\
\text { und Risiko- } \\
\text { gruppen }\end{array}$ & $\begin{array}{l}\text { Unterstützung von Gesund- } \\
\text { heitsadministrationen unter } \\
\text { Berücksichtigung Basis- } \\
\text { gesundheitsprinzipien, ins- } \\
\text { besondere der Gemeinde- } \\
\text { beteiligung und der intersek- } \\
\text { toriellen Zusammenarbeit, } \\
\text { Technische Assistenz }\end{array}$ & $\begin{array}{l}\text { Regisseure der } \\
\text { schweizerischen } \\
\text { Entwicklungszusam- } \\
\text { menarbeit } \\
\text { Partnerinstitutionen }\end{array}$ & $\begin{array}{l}\text { Nichtstaatliche und } \\
\text { staatliche Institutionen } \\
\text { in der Schweiz und } \\
\text { Partnerländer, mittlerer } \\
\text { bis sehr hoher Finanz- } \\
\text { mittelbedarf }\end{array}$ \\
\hline $\begin{array}{l}\text { Distrikts- } \\
\text { Gesundheits- } \\
\text { system- } \\
\text { Forschung }\end{array}$ & $\begin{array}{l}\text { abhängig von } \\
\text { Forschungsthemen, } \\
\text { von klar umschrie- } \\
\text { benen bis zur } \\
\text { allgemeinen } \\
\text { Bevölkerung }\end{array}$ & $\begin{array}{l}\text { abhängig von } \\
\text { Forschungsthemen, } \\
\text { aber oft direkt auf } \\
\text { die Bevölkerung } \\
\text { bezogen }\end{array}$ & $\begin{array}{l}\text { Angewandte Gesundheits- } \\
\text { systemforschung, die in einem } \\
\text { partizipativen Ansatz natur- } \\
\text { und sozialwissenschaftliche } \\
\text { Techniken miteinander ver- } \\
\text { bindet / Technische Assistenz }\end{array}$ & $\begin{array}{l}\text { v.a. } \\
\text { Forschungsinstitutionen in } \\
\text { der Schweiz, den Partner- } \\
\text { ländern und Forschungs- } \\
\text { partner aus der nördlichen } \\
\text { Hemisphäre }\end{array}$ & $\begin{array}{l}\text { Nichtstaatliche und } \\
\text { staatliche Institutionen } \\
\text { in der Schweiz und } \\
\text { Partnerländer, mittlerer } \\
\text { bis hoher Finanzmittel- } \\
\text { bedarf }\end{array}$ \\
\hline $\begin{array}{l}\text { Regionale und } \\
\text { internationale } \\
\text { Unterstützung }\end{array}$ & $\begin{array}{l}\text { Institutionen oder } \\
\text { Organisationen }\end{array}$ & International & $\begin{array}{l}\text { Finanzielle Unterstützung / } \\
\text { Technische Assistenz }\end{array}$ & $\begin{array}{l}\text { Direktion für Entwicklungs- } \\
\text { zusammenarbeit und hu- } \\
\text { manitäre Hilfe / Schweize- } \\
\text { risches Forschungsinstitu- } \\
\text { tionen / Medicus Mundi }\end{array}$ & $\begin{array}{l}\text { Staatliche institutionen } \\
\text { in der Schweiz und in } \\
\text { Partnerländern, sehr } \\
\text { hoher Finanzmittelbedarf }\end{array}$ \\
\hline
\end{tabular}


müssen. Bei Komplikationen und der Abwesenheit einer in der Geburtshilfe erfahrenen Person kann dies zu bedrohlichen Verzögerungen bei der Einleitung notwendiger Therapiemassnahmen führen. Um dieses Problem zu lösen, unterstützt die schweizerische Ostasienmission die Ausbildung von Dorfhebammen, sogenannten "Mama Biang". Diese Frauen, die aus den Dorfgemeinschaften stammen, werden ausgebildet, eine normale Geburt adäquat zu betreuen und rechtzeitig allfällig auftretende Komplikationen zu erkennen. Diese Frauen erhalten darüberhinaus eine Ausbildung in präventiven Massnahmen, die helfen sollen, den Gesundheitszustand von Müttern und Kindern zu verbessern. Die Auswahl und Ausbildung der Frauen erfolgt unter direkter Beteiligung der Dorfbevölkerung. Diese Massnahmen in Tomohon begannen schon 1973 und haben bis 1991 zur Ausbildung von über 200 Dorfhebammen geführt. Zusammen mit der kontinuierlichen, fachlichen Betreuung der Dorfhebammen und einem gut funktionierenden Referenzsystem hat diese Basismassnahme zu einer wesentlich verbesserten geburtshilflichen Versorgung auf dem Inselarchipel geführt.

Die Beispiele zeigen den Basisansatz der Massnahmen, die direkt eine mehr oder weniger genau definierte, auf jeden Fall aber überschaubare Zielgruppe haben. Die Erfolge dieser Projekte sind deutlich sichtbar und haben zur Verbesserung der Lebenssituation der Zielgruppen geführt. Ein wichtiges Charakteristikum dieses Ansatzes ist, dass die Projekte nur marginal, wenn überhaupt, Kontakt mit staatlichen Strukturen haben. Das Referenzspital in Tomohon ist beispielsweise auch ein kirchlich geleitete Krankenhaus ohne grossen Kontakt zu den staatlichen Strukturen. In beiden Projekten wird das geringe Interesse und die daraus resultierende geringe Unterstützung der staatlichen Gesundheitsstrukturen beklagt. Die Gründe hierfür sind vielschichtig. Zum einen ist die öffentliche Verwaltung in vielen Ländern schwach. Dies erklärt sich einerseits durch Mittelknappheit aber auch durch Ausbildungs- und Erfahrungsdefizite. Das führt zu inkoheränten Planungen, zu bürokratischen, manchmal ineffizienten Abwicklungen, die der Dynamik von Basisaktionen nicht genügend Spielraum lassen. Es gibt aber auch Beispiele, die zeigen, dass die Integration und Zusammenarbeit mit staatlichen Einrichtungen durchaus möglich ist.

Im Rahmen eines tansanisch-schweizerischen Projektes zur Verbesserung der städtischen Gesundheitsversorgung in Dar es Salaam wird beispielsweise versucht, Frauenselbsthilfegruppen zu organisieren und durch kleine gemeindegetragene Ausbildungen dafür zu sorgen, dass die Frauen kleine einkommensschaffende Tätigkeiten entwickeln können. Durch eine partnerschaftliche Diskussion mit einer 140 Frauen umfassenden Gruppe wurden Möglichkeiten zur Verbesserung der Lebensqualität in einem Vorort von Dar es Salaam diskutiert und von der Frauengruppe ein Aktionsplan entwickelt. Dieser sieht eine Serie von Aktivitäten vor, die zur Reduktion der Häufigkeit von Malariaerkrankungen führen soll. Als nächstes Problem hat die Frauengruppe damit begonnen, einkommensschaffende Aktivitäten, wie beispielsweise die Herstellung von Strickwaren, durchzuführen. Die externe Unterstützung besteht im wesentlichen in der Stimulation der Gruppe, sich selbst zu organisieren, Probleme selbst zu erkennen und gemeinsam anzugehen. 
Ein anderes Beispiel ist das weiter unten beschriebene Projekt der DEH in Nepal, das wesentliche basisbezogene Zielsetzungen, insbesondere bezüglich der natürlichen Familienplanung, beinhaltet.

Bei dieser Art von Projekten ist der gesamthafte Ansatz hervorzuheben, der versucht, auf der Ebene von Familien oder klar umschriebenen, oft relativ kleinen Gemeinden Lösungen für verschiedene, über die reine Gesundheitsproblematik hinausgehende Problemkreise zu finden. Wesentlich ist auch, dass in vielen Fällen die Initiative zu einer Unterstützung nicht von aussen herangetragen wird, sondern in einer lokalen Bedürfnisformulierung ihre Ursprünge hat. Der Grundgedanke einer Partnerschaft ist oft ebenfalls verwirklicht, da die Betroffenen ein wesentliches Mitspracherecht bei der Formulierung von Zielen und deren Umsetzung besitzen.

Ein grundsätzliches Problem des basisnahen Ansatzes ist die begrenzte Übertragbarkeit auf andere Situationen. Diese hängt aber auch mit der politischen Aussage zusammen, die mit dem Konzept der basisnahen Aktivitäten verbunden ist, und die in vielen Situationen beinahe revolutionäre Züge trägt. Der Basisansatz hat letztendlich eine sehr politische Dimension, in dem er die Eigenverantwortlichkeit der Bevölkerung fördert. Diese wird, wenn sie erfolgreich ist, kaum auf die Gesundheit beschränkt bleiben, sondern dazu führen, dass auch für andere Bereiche die Verantwortlichkeit eingefordert wird. Sehr schnell werden dadurch herrschende Machtstrukturen in Frage gestellt, weshalb oft Widerstände von staatlichen Stellen zu verzeichnen sind.

\section{B) Unterstützung von Gesundheitseinrichtungen}

Dieser Ansatz der Entwicklungszusammenarbeit hat seine Wurzeln in der kirchlichen Missionsarbeit und ist bis heute ein Schwerpunkt der Arbeit von schweizerischen Nichtregierungsorganisationen geblieben.

Die Basler Mission ergänzte seit 1831 ihre Mission mit Gesundheitsarbeit an der Goldküste, dem heutigen Ghana. Auch wenn es Ansätze zu einem integrierten Gesundheitssystemansatz gab (s. Abschnitt C), lag der Schwerpunkt der Arbeit auf der kurativen Medizin. Beispiele hierfür sind die bis heute existierenden Missionsspitäler in Lesotho, Zimbabwe, Ghana und Tansania.

Ein verhältnismässig neuer Ansatz sind Partnerschaften zwischen Schweizer Spitälern und solchen in Entwicklungsländern. Beispiele hierfür ist die Arbeit von Jura-Afrique, die ein Krankenhaus im Norden Benins unterstützt. Jura-Afrique zeigt, dass, obwohl die Eintrittspforte das Krankenhaus ist, auch periphere Aktivitäten, bspw. die Ausbildung von Dorfgesundheitshelfern in die Unterstützung integrierbar sind. Ansätze der peripheren Unterstützung finden sich heute - wenn auch mit teilweise sehr unterschiedlicher Gewichtung - in fast allen oben erwähnten Missionsspitälern.

Ein anderes Beispiel ist die Organisation Fondation de Jumelage de l'Hôpital du Samaritain, die ein Spital in Kamerun unterstützt. Bei dieser Hilfe steht die 
Versorgung mit medizinischem Material im Vordergrund und beschränkt sich auf das Jumelagespital.

Wie das Beispiel einer von der Schweiz ausgehenden, privaten Unterstützungsintitiative eines Kinderspitals in Phnom Penh zeigt, besteht in der Schweiz immer noch ein enormes Potential, Spendengelder für isolierte Krankenhausprojekte zu mobilisieren. Dieses Projekt zeigt exemplarisch die Probleme auf, die bei der Integration in bestehende Strukturen entstehen können und die Bedingungen für die Nachhaltigkeit des Projekts. Beide Problemkreise sind von grundsätzlicher Bedeutung, wenn es um die langfristige Überlebensfähigkeit einer derartigen Einrichtung geht, die irgendwann einmal von der externen Unterstützung unabhängig werden muss. Bedauerlicherweise scheinen derartigen Überlegungen bei der Konzeption dieses Hilfsprojektes nur eine sekundäre Bedeutung beigemessen worden zu sein. Alternativen für dieses mit enormen Investitionen und Unterhaltskosten verbundene Projekt scheinen auch nicht diskutiert worden zu sein, da die "Lösung", d.h. Spital, schon vor der Analyse der vorhandenen Probleme festgestanden hat. Das Schweizerische Rote Kreuz zeigt ebenfalls in Kambodscha seit 1986 mit der Unterstützung des Provinzspitals Takéo, dass humanitäre Unterstützung eine sinnvolle Planung und Integration in bestehende Strukturen nicht ausschliessen muss. Das vom Schweizerischen Roten Kreuz unterstützte Spital und seine peripheren Kliniken sichern die Gesundheitsversorgung einer ganzen Provinz mit einer Bevölkerung von etwa 700'000 Menschen. Es ist gelungen, mit dem Provinzspital ein den lokalen Verhältnissen angepasstes Versorgungszentrum aufzubauen. Der Prozess der Übergabe an einheimische Fachkräfte hat begonnen.

Die Evolution von einem rein kurativen Spitalprojekt zu einem integrierten Bestandteil eines Distriktgesundheitssystems kann auch an der Entwicklung der Unterstützung des Zentralspitals N'Djaména im Tschad aufgezeigt werden. Die schweizerische Unterstützung begann in den späten siebziger Jahren als reines Krankenhausprojekt. Die Funktion einer allgemeinmedizinischen Abteilung des Zentralkrankenhauses hat sich über die Jahre gewandelt und wurde neu definiert. Heute erfüllt diese Abteilung die Aufgaben eines Distriktkrankenhauses für einen Teil der Stadt. Die von der DEH finanzierte technische Assistenz entwickelte sich von der direkten medizinischen Betreuung von Kranken zur Unterstützung der Krankenhausverwaltung, mit einem besonderen Schwerpunkt auf der Fort- und Weiterbildung des Personals.

Der Überblick über diese Aktivitäten zeigt, dass die reine Krankenhausmedizin von den meisten schweizerischen Organisationen und Institutionen aufgegeben worden ist und fast überall der Versuch unternommen wird, nicht nur Krankenhäuser sondern auch die peripheren Gesundheitseinrichtungen bis hin zu gemeindegestützten Aktivitäten zu stärken.

Hervorzuhebende Erfahrungen sind im Bereich der standardisierten Behandlung von Krankheitsbildern, wie sie beispielsweise in Tansania, im St. Francis Designated Distrikt Hospital entwickelt wurden, oder aber auch Erfahrungen mit Finanzierungssystemen, wie sie in von der Schweiz (Solidarmed) unterstützten Missionsspitälern in Zimbabwe gemacht worden sind. Auch im Bereich einer den 
Bedürfnissen angepassten Fortbildung im Spitalumfeld wurden und werden wertvolle Erfahrungen gemacht. Ein grundsätzliches Problem stellt die Integration dieser Spitäler in die jeweiligen staatlichen Gesundheitssysteme dar. Das St. Francis Designated Distrikt Hospital, das von Solidarmed unterstützt wird, sollte schon vor Jahren in das tansanische Gesundheitssystem integriert werden. Die tansanische Regierung hat diese Integration und damit die Übernahme der Verantwortlichkeit für dieses Krankenhaus nie übernommen und im Gegenteil von diesen Plänen Abstand genommen. Einer der Gründe hierfür mag in den relativ hohen laufenden Kosten und im kontinuierlichen Investitionsbedarf liegen, die mit einem Spitalbetrieb verbunden sind, und der tansanische Staat wohl zu Recht befürchtet, diese nicht aufbringen zu können.

Schon Anfang dieses Jahrhunderts erkannte der Arzt Dr. Fisch (Fischer (4), 1991) von der Basler Mission in Ghana, dass die rein kurative Medizin, wie er sie in seinem Krankenhaus praktizierte, nicht die ganze Antwort auf die herrschenden Gesundheitsprobleme sein konnte. Er setzte sich deshalb für eine Dezentralisierung der Gesundheitsdienstleistungen ein und nahm Elemente der Gesundheitserziehung in seine Arbeit auf. Auch der traditionellen Medizin widmete er sein Interesse. Er war damit ein Vorreiter des heutigen ganzheitlichen Ansatzes der dezentralen Gesundheitsversorgung. Bei diesem ganzheitlichen Ansatz stellt sich immer die Frage der Gewichtung der Rolle des Krankenhauses im besonderen, bzw. von Gesundheitseinrichtungen im allgemeinen. Auf Grund der Entwicklung haben Krankenhäuser fast immer die Tendenz, durch die ihnen eigene Dynamik und ihren Ressourcenbedarf ein relatives Übergewicht im Vergleich zu peripheren Aktivitäten zu erlangen. Aus diesem Grund sind Versuche, "Gesundheit" aus den Gesundheitseinrichtungen heraus in die Peripherie zu tragen, fast immer mit einer Hypothek belastet, da es - nicht nur in der südlichen Hemisphäre - den für Gesundheitseinrichtungen Verantwortlichen schwer fällt, Ressourcen umzuverteilen. Ein Ansatz dies zu erreichen ist es, Gesundheitseinrichtungen nicht isoliert, sondern als Teil eines Distriktsgesundheitssystems zu sehen. In der Praxis bedeutet dies, die Unterstützung nicht direkt via bestehende Gesundheitseinrichtungen den Partnerländern zukommen zu lassen, sondern durch die - meistens staatliche - Gesundheitsverwaltung.

\section{C) Gesamthafte Unterstützung von Distriktsgesundheitssystemen}

Die als private Initiative begonnene und später von der DEH unterstützte "Acción médica Suiza Puno" in Peru setzte bereits Anfang 1970 neue Massstäbe in der medizinischen Entwicklungszusammenarbeit (Cadotsch (5), 1977). Präventive Aspekte waren in diesem Projekt genauso wichtig wie die kurative Medizin. Die Verbesserung der Medikamentenversorgung und der Finanzierung der Gesundheitsdienste waren Schwerpunkte der Projektaktivitäten. Grosse Anstrengungen wurden unternommen, die Bevölkerung in diese Aktivitäten miteinzubeziehen.

Das von der DEH unterstützte Integrated Hill Development Project in Nepal war international eines der ersten regionalen Entwicklungsprojekte in dem verschie- 
dene Sektoren, von der Landwirtschaft, Aufforstung bis zur Gesundheit integriert unterstützt wurden. Seit Ende der siebziger Jahre war in dem grossen Projekt auch eine bedeutende Gesundheitskomponente enthalten. Dieses Projekt war damit international eines der ersten ländlichen Entwicklungsprojekte, das die Bedeutung des Gesundheitssektors für einen gesamthaften Entwicklungsprozess erkannte und umsetzte. Der Beitrag bestand in der Unterstützung der Distriktsgesundheitsverwaltung (District Health Inspectors). Schwerpunkte der Tätigkeit waren die Medikamentenversorgung, der Bau von Dispensarien, die Ausbildung von Dorfgesundheitshelfern (Community Health Leaders) und Dorfhebammen (Traditional Birth Attendants). Auch die Familienplanung, insbesondere die Verbreitung natürlicher Methoden, waren wichtige und richtungsweisende Aktivitäten. Ein Latrinenbauprogramm sowie die Promotion von raucharmen Öfen waren flankierende Massnahmen, in die sich bspw. auch die Verbesserung der Trinkwasserversorgung einordnete. Bei der Medikamentenversorgung wurden schon damals Überlegungen der Kostenbeteiligung der Patienten umgesetzt. Allerdings konnte kaum die Hälfte der Behandlungskosten durch die lokalen Einnahmen gedeckt werden. Hervorstechendes Merkmal der breit angelegten Unterstützung des Gesamtprojektes war die sehr weitgehende Integration in die nationalen Strukturen. Dies war aber auch eine der Hauptschwierigkeiten, in dem es zu enormen Problemen bei der Koordination der verschiedenen Projektkomponenten kam. Das Integrated Hill Development Project wurde in der Zwischenzeit in drei kleinere, aber immer noch eng koordinierte, Sektorprojekte aufgeteilt. In der Gesundheitskomponente wird neben der fortgesetzten Unterstützung der Gesundheitsverwaltung besonderer Wert auf die Basisarbeit mit Frauengruppen Wert gelegt.

Im Auftrag der DEH implementiert das IUED seit 1981 mit dem Beniner Gesundheitsministerium das "Programme Medico-Sanitaire Benino-Suisse" in Distrikten im Süden Benins. Schwerpunkte der Aktivitäten waren der Aufbau einer Gesundheitsdienstleistungsinfrastruktur und die Unterstützung der Gesundheitsverwaltungen. Dabei fand der Basisgesundheitsansatz, insbesondere die Gemeindebeteiligung in Form von Ausbildung und Supervision von Dorfgesundheitshelfern, besondere Beachtung. Die Medikamentenversorgung wurde durch die Einführung einer Kostenbeteiligung der Patienten sichergestellt. Die Mutter-Kindbetreuung, insbesondere die Erhöhung der Impfdeckung, wurde gestärkt. Die Fortund Weiterbildung des medizinischen und paramedizinischen Personal hat auf sämtlichen Ebenen des Projektes eine zentrale Bedeutung. Der Bau von Tiefbrunnen und Abwasserentsorgungssystemen erfolgte komplementär zu den reinen Gesundheitssektorinterventionen.

Ebenfalls im Auftrag der DEH ist das IUED in einem weiteren, ähnlich konzipierten Projekt in Mali aktiv. Neben den oben erwähnten Elementen ist für dieses Projekt der Planungsaspekt ein wesentlicher Bestandteil. Im Distrikt Sikasso wurde als einem der ersten Distrikte in Mali ein Gesundheitsplan erstellt, der nicht nur für das Projekt selbst, sondern auch für andere Interventionen im Gesundheitsbereich eine Planungsgrundlage gebildet hat.

Die DEH unterstützt ausserdem in bilateralen Projekten Distriktsgesundheitssysteme in Tansania und im Tschad. Im Tschad konzentriert sich die Aktivität auf 
die Unterstützung der Tschader Gesundheitsbehörden im Aufbau eines funktionalen Gesundheitssystems in den Präfekturen Chari-Baguirmi und Moyen-Chari sowie in der Hauptstadt N'Djaména. Die Aktionsebene ist auch hier der Distrikt, und die schweizerischen Projekte unterstützen die allgemeine Gesundheitsadministration, besonders bei der Planung und der Verbesserung der Medikamentenversorgung. Bei letzterer werden vor allem Aspekte der Beschaffung, Lagerung, Verteilung aber auch der Anwendung berücksichtigt.

Eine wichtige Leistung des schweizerischen Beitrags im Tschad war die Definition eines "Paquet minimal" der Dienstleistungen, die in einer Gesundheitseinrichtung erbracht werden müssen. Derartige Standards, die sowohl kurativmedizinische als auch präventivmedizinische Aspekte umfassen, sind eine wesentliche Voraussetzung bei der Verbesserung von Gesundheitsdiensten, weil erst dadurch die sinnvolle, den lokalen Verhältnissen angepasste Beschaffung von Ausstattungsmaterial möglich wird. Im Hinblick auf die Nachhaltigkeit bildet ein derartiges "Paquet minimal" die Grundlage für eine konstruktive Supervision und damit Qualitätssicherung der Dienstleistungen. Für die Umsetzung dieser Projekte wurden so in den vergangenen Jahren knapp sieben Millionen Schweizer Franken aufgewendet. Dies scheint ein hoher Betrag zu sein. Man muss allerdings berücksichtigen, dass mit dieser Massnahme einer Bevölkerung von einer halben Million eine wesentliche Verbesserung in der Verfügbarkeit von angepassten Gesundheitsdiensten erreicht wurde. Die Schaffung eines funktionierenden Referenzsystems hat ebenfalls eine grosse Bedeutung (s.a. Teil "B", Zentralkrankenhaus von N'Djaména). Im Nordosten des Tschad unterstützt das Schweizerische Rote Kreuz (mit einer Teilfinanzierung der DEH) den Distrikt Biltine mit seinem Distriktskrankenhaus und den dazugehörenden Aussenstationen. Die Durchführung dieses Projekts findet unter enger Koordination mit den anderen Aktivitäten der schweizerischen Entwicklungszusammenarbeit im Gesundheitssektor statt. In der Hauptstadt N'Djaména stellt das STI im Rahmen eines multilateralen Projektes zur Verbesserung der städtischen Gesundheitsversorgung die technische Expertise mit zwei Expertinnen zur Verfügung. Schwerpunkt im Aufbau eines dezentralisierten, städtischen Gesundheitssystems sind neben den für die beiden oben erwähnten planerischen und verwaltungstechnischen Aspekten die Anstrengungen, die Gemeindebeteiligung zu fördern und ein Kostenbeteiligungsmodell einzuführen.

In Dar es Salaam, der bald zwei Millionen Einwohner zählenden Hauptstadt Tansanias unterstützt die DEH ein breit angelegtes Projekt zur Verbesserung der städtischen Gesundheitsversorgung. Die Aktionsebene ist auch hier der Distrikt. Die Ziele lassen sich in drei Kategorien aufteilen. Zum einen soll das öffentliche Gesundheitssystem in den Distrikten funktionaler werden. Dies beinhaltet neben infrastrukturellen Massnahmen wie die Renovation und den Neubau von drei Distriktskrankenhäusern, vier Gesundheitszentren und über fünfzig Dispensarien vor allem die qualitative Verbesserung des Gesundheitssystems. Dabei spielen sowohl Aspekte der Finanzierung wie auch der Qualitätsverbesserung der eigentlichen medizinischen Leistungen eine wesentliche Rolle. Ein zweiter, wichtiger Schwerpunkt der Projektaktivitäten ist die Förderung der Gemeindebeteiligung 
bzw. deren Einbeziehung. Besonders Frauen als benachteiligte Bevölkerungsgruppe wird versucht, durch Selbsthilfegruppen mit Rat und Tat zur Seite zu stehen. Ein dritter Schwerpunkt des Projektes ist die Ausarbeitung von Konzepten zur städtischen Gesundheitsversorgung in Tansania. Derartige Konzepte, insbesondere auch was die Einbeziehung der Bevölkerung angeht, fehlen nicht nur in Tansania. Die wesentlichen Komponenten aller schweizerischen Projekte, die einen gesamthaften Ansatz der Distriktsgesundheitsversorgung haben, lassen sich wie folgt zusammenfassen:

- Stärkung der Managementkapazitäten der Distriktsgesundheitsadministration, um die Nutzung knapper Ressourcen zu optimieren;

- Unterstützung bei notwendigen Ausbildungsmassnahmen (s.a. entsprechendes Kapitel);

- Unterstützung bei Konzepten und Umsetzung von Gemeindebeteiligung bei Aktionen im Gesundheitsbereich;

- Stimulation von lokalen Partnern, diese Konzepte aufzunehmen und umzusetzen;

- Lokalen Standards angepassste Renovation der baulichen Infrastruktur und entsprechende Ausstattung mit medizinischen Gerätschaften;

- Stärkung des Dezentralisierungsprozesses, insbesondere was Entscheidungs- und Verfügungsgewalt im Gesundheitssystem angeht;

- Die Koordination der Aktivitäten in einem Distriktsgesundheitssystem, indem die Entwicklung funktioneller Kommunikationsprozesse zwischen dem Gesundheitssektor und anderen Sektoren wie Landwirtschaft und Erziehung, die zur Verbesserung des Gesundheitszustandes einer Bevölkerung beitragen können, gefördert wird;

- Die Versorgung mit essentiellen Medikamenten;

- Finanzierungaspekte von Distriktsgesundheitssystemen;

- Die Miteinbeziehung und Koordination von nichtstaatlichen Gesundheitsanbietern, insbesondere auch von privaten, profitorientierten Anbietern bei der Planung eines Distriktsgesundheitssystems.

Die Hauptprobleme bei der Umsetzung dieses Ansatzes liegen in der Dezentralisierung, in der dauerhaften Finanzierung der Gesundheitssysteme und in der Einbeziehung der Bevölkerung in die Aktivitäten.

Die Dezentralisierung von Verantwortlichkeit in den Gesundheitsverwaltungen trifft in vielen Entwicklungsländern auf Widerstand. Seit ihrer Unabhängigkeit sind oft sehr zentralistische Verwaltungsstrukturen aufgebaut worden, die schwerfällig und wenig effizient auf lokale Bedürfnisse und Gegebenheiten reagieren können. Neben dem politischen Willen der zentralen Strukturen, wie Gesundheitsministerien, Verantwortung und damit Kontrolle abzugeben, fehlt es oft aber auch an erfahrenen Distriktsverantwortlichen, die es nicht gewohnt sind, selbständig Entscheidungen zu treffen und diese zu verantworten. Ein Grundproblem ist auch die Ressourcenknappheit auf der Distriktsebene. Nach der Unabhängigkeit vieler Staaten, v.a. im südlichen Afrika, hatten die jungen Staaten die Absicht, gewisse 
Grundbedürfnisse der Bevölkerung, insbesondere Gesundheits- und Erziehungsdienstleistungen, kostenlos anzubieten. Die damit verbundenen Kosten und die allgemeine Mittelknappheit haben dazu geführt, dass die meisten Länder diesen Anspruch heute nicht mehr erfüllen können. Die Finanzierung von Gesundheitssystemen wurde deshalb von sehr grosser Bedeutung.

Die aktive Miteinbeziehung der betroffenen Bevölkerung ist ein weiterer zentraler Punkt der Zusammenarbeit und eine Grundvoraussetzung für eine nachhaltige Entwicklung, nicht nur im Gesundheitsbereich. Die gesamthaften Ansätze versuchen Konzepte der aktiven Miteinbeziehung der Bevölkerung zu integrieren, wobei hier die Schwierigkeiten, die es schon auf der Basisebene gibt, durch die Zahl und natürliche Heterogenität der Bevölkerung und deren Interessen auf der Ebene eines Distrikts erschwert ist. In diesen Bereichen fehlt es nicht an Konzepten, aber es besteht noch ein grosser Bedarf, diese den lokalen Verhältnissen anzupassen. Der Bedarf für eine wissenschaftliche Basis für Interventionen auf Distriktsebene ist daher gross.

Die Schweiz (IUED/ASSN/DEH (6), 1992) hat in diesem Bereich Beachtenswertes geleistet.

\section{D) Auf den Distrikt ausgerichtete Gesundheitssystemforschung}

Das STI und das IUED sind international anerkannte Forschungseinrichtungen. Partnerschaften mit Forschungsinstitutionen sowohl im Süden als auch im Norden und eine Vielzahl von wissenschaftlichen Publikationen in Bereich Distriktsgesundheitssystemforschung belegen dies.

Das STI hat 1957 in Ifakara/Tansania ein Feld-Laboratorium gegründet, das in seinen Anfängen vor allem biomedizinische Grundlagenforschung betrieben hat. Seit 1982 hat das Zentrum sein Forschungsspektrum durch die Distriktsgesundheitssystemforschung erweitert (Tanner (7) et al, 1993). Die Themen, die bearbeitet wurden, sind breitgefächert. Bei den Arbeiten in Ifakara ging es bis heute nicht nur darum in bezug auf Diagnose, Therapie und Prophylaxe neue Ergebnisse zu erzielen, sondern Ziel war bei allen Aktivitäten die Umsetzung dieser Ergebnisse im Rahmen einer distriktgestützten Basisgesundheitsversorgung, um den grösstmöglichen Nutzen für die Bevölkerung zu erreichen. Vor allem operationelle Aspekte, wie beispielsweise die Beteiligung der Bevölkerung bei präventiven Gesundheitsmassnahmen, oder die optimale Ausbildung von Gemeindegesundheitshelfern, sowie die regelmässige Evaluierung standen und stehen im Vordergrund. In dem Ifakara Centre wurden auch einfache Methoden zur Identifikation von Dörfern entwickelt, in denen die Blasenbilharziose ein grosses Problem darstellt. Diese Methoden können auch von Distriktsärzten, die nur über begrenzte Ressourcen verfügen, eingesetzt werden. Eine weitere wichtige Arbeit, die in den letzten Jahren in Ifakara geleistet wurde, beschäftigte sich mit gesundheitsökonomischen Fragestellungen und dem Problem der Qualität von Dienstleistungen in einem Gesundheitsdistriktsystem. Ein Ergebnis waren beispielsweise auffallende Unterschiede in den Personalkosten pro Patient. Die Resultate der For- 
schungsarbeiten konnten teilweise in dem zugehörigen Distrikt umgesetzt werden, darüber hinaus fanden diese Arbeiten aber auch auf nationaler und internationaler Ebene ein breites Echo und grosse Akzeptanz. Die für die Bekämpfung der Bilharziose entwickelten Instrumente (Lengeler (8)) konnten in einem von der Weltgesundheitsorganisation unterstützten Vergleich in sieben weitere afrikanische Länder (Äthiopien, Kamerun, Kongo, Malawi, Zaïre, Zambia, Zimbabwe) übertragen werden, und es konnte gezeigt werden, dass diese Instrumente sich auch für andere Tropenkrankheiten anwenden lassen. Ferner wurde und wird eine Vielzahl von Untersuchungen und Studien zur Kontrolle übertragbarer Erkrankungen durchgeführt. Die wesentlichste Aktivität in diesem Bereich ist die Testung der Wirksamkeit eines Impfstoffes gegen die Malaria (Teuscher (9) et al, 1994), der von dem kolumbianischen Immunologen Manuel Patarroyo entwickelt worden ist. Dieser hat auch erste vielversprechende Untersuchungen in Lateinamerika durchgeführt hat. Bei der Untersuchung in Tansania, die zusammen mit dem tansanischen National Institut of Medical Research, dem spanischen Forschungsrat, der London School of Hygiene and Tropical Medicine mit der Unterstützung der Weltgesundheitsorganisation durchgeführt wird, handelt es sich aber um den weltweit ersten Versuch in einem Gebiet mit einer hohen Übertragungsrate der Malaria. Nicht nur die wissenschaftliche Fachwelt erwartet die Ergebnisse dieser vielbeachteten Studie mit Spannung, da auf Distriktsebene eine dringend benötigte Ergänzung der Möglichkeiten der Malariabekämpfung zur Verfügung stehen würde, falls der Nachweis gelingen sollte, dass der Impfstoff die Häufigkeit der Malariaerkrankungen verringern kann.

Die aktive und eigenverantwortliche Beteiligung der Bevölkerung an diesen Untersuchungen ist ein Grundzug der wissenschaftlichen Arbeit am Forschungszentrum in Ifakara, das sich im Augenblick auf dem Weg der vollständigen Integration in die nationale Forschungsinstitution (National Institute of Medical Research) befindet. In Dar es Salaam wurden im Rahmen eines von der DEH unterstützten städtischen Gesundheitsprojekts (in Zusammenarbeit mit Forschern der Universität von Dar es Salaam und der London School of Hygiene and Tropical Medicine), Untersuchungen über die Qualität der Gesundheitsdienstleistungen in staatlichen und Missionsgesundheitseinrichtungen durchgeführt. Die Ergebnisse zeigten, dass sich die Patienten in den Missionseinrichtungen besser behandelt fühlten. Rein medizinisch war aber die Qualität in beiden Arten durchaus vergleichbar. Die Ergebnisse dieser und anderer Studien tragen dazu bei, neue und innovative Konzepte zu entwickeln, um die mit der Verstädterung in den Entwicklungsländern einhergehenden Gesundheitsprobleme zu bewältigen.

Das IUED führt im Rahmen seiner Projekte in Benin und Mali ebenfalls projektbegleitende und unterstützende Forschungen durch. Diese haben durch epidemiologische Untersuchungen über vorherrschende Gesundheitsprobleme und über den Ernährungszustand von Kindern dazu beigetragen, die Planung in den von den Projekten unterstützten Gesundheitsdistrikten kohärenter und konsistenter werden zu lassen (Izurieta, Do Rego (10) et al., 1990). Arbeiten über die lokale Finanzierung der Gesundheitssysteme wurden vom IUED in Cotonou und Bamako durchgeführt. 
Angewandte Forschung wird aber auch von anderen schweizerischen Organisationen unterstützt. Medicus Mundi Schweiz finanziert zusammen mit der DEH, der Rudolf-Geigy-Foundation und der Stanley Thomas Johnson Foundation in Zimbabwe eine Untersuchung über die Auswirkungen von Sanitärmassnahmen auf Distriktsebene auf den Gesundheitszustand der Bevölkerung, insbesondere die Reduzierung von Durchfallerkrankungen. Die Gruppe für Entwicklungsarbeit Basel (GEAB) unterstützt angewandte Forschung in Nicaragua, um angepasste Gesundheitserziehung zu definieren, welche die Übertragung des HI-Virus bei Sexarbeiterinnen reduzieren helfen soll.

In den Lehraktivitäten des STI spielt der Distriktschwerpunkt eine grosse Rolle, und in der praxisorientierten Ausbildung des "Health Care and Management in Tropical Countries" ist der Gesundheitsdistrikt der Bezugspunkt der Lehrinhalte. In diesem Zusammenhang wurde ein computergestütztes Modell entwickelt, mit dem für Lehrzwecke Gesundheitsinterventionen und ihr Einfluss auf den Gesundheitszustand einer Bevölkerung simuliert werden können. Dieses Modell fusst auf Daten aus dem Kilombero Distrikt in Tanzania. Im Augenblick wird das Modell aber auch auf Distrikte in anderen Ländern, insbesondere Thailand und Indien, angepasst. Im Augenblick ist es ausschliesslich für Lehrzwecke geeignet. Denkbar ist aber, dass es in der nahen Zukunft auch für Planungszwecke eingesetzt werden kann. Eine besondere, distriktsorientierte Fragestellung wird vom STI in Zusammenarbeit mit Forschungseinrichtungen aus Congo, Belgien, Deutschland, Marokko und Tschad bearbeitet. Es geht bei diesem von der Europäischen Gemeinschaft und der Schweizerischen Kommisssion zur Förderung der wissenschaftlichen Forschung finanzierten Forschungsvorhaben darum, die Kommunikation zwischen verschiedenen Versorgungsstufen in einem Distriktsgesundheitssystem zu untersuchen und Verbesserungsvorschläge zu erarbeiten. Grundgedanke ist, dass es trotz aller offensichtlichen Verschiedenheiten auch Gemeinsamkeiten zwischen einem Gesundheitsdistrikt in der Schweiz und beispielsweise einem Distrikt im Tschad gibt. Von dieser Annahme aussgehend können gemeinsame Lösungen gefunden werden.

Diese Zusammenarbeit von Schweizer Forschungsinstitutionen (Swiss Tropical Institute (11), 1993) und Partnereinrichtungen aus der südlichen Hemisphäre hat im Bereich der Distriktsgesundheitssystemforschung zu einer gemeinsamen Entwicklung und gegenseitigen Stimulation und vor allem zu einer an den Bedürfnissen orientierten Wissenschaft geführt. Dies ist im internationalen Vergleich seltenes Beispiel einer partnerschaftlichen Entwicklungszusammenarbeit im Wissenschaftsbereich. Im Vergleich zu anderen europäischen Forschungseinrichtungen, die auf dem Gebiet der Gesundheitssystemforschung tätig sind, haben das IUED und das STI die Besonderheit, dass sie nicht "nur" forschen, sondern auch direkte Verantwortung für die Umsetzung von Projekten der Entwicklungszusammenarbeit besitzen. Die Herausforderung der Kombination von Forschung und Umsetzung wird von der DEH und dem Schweizerischen Nationalfonds durch ein spezielles Schwerpunktprogramm Umwelttechnologie und Umweltforschung (Modul 7) aufgenommen, mit dem für die Umwelt in der südlichen Hemisphäre relevante Themen, insbesondere auch deren Bedeutung für die Gesundheit, 
bearbeitet werden sollen. Eher allgemeiner Natur, aber Gesundheitsaspekte einschliessend, ist die Initiative der Schweizerischen Akademie der Naturwissenschaften, mit der die praxisorientierte Forschungszusammenarbeit mit Entwicklungsländern gefördert werden soll. Wie die oben erwähnten Beispiele zeigen, hat diese Verbindung mit der Praxis sichergestellt, dass sich die Forschungsthemen an den Bedürfnissen der Partnerländer orientieren.

\section{E) Unterstützung des Distriktskonzepts auf regionaler, nationaler und internationaler Ebene}

Medicus Mundi Schweiz fördert als Dachorganisation der wichtigsten im Gesundheitssektor aktiven Entwicklungsorganisationen und Institutionen in der Schweiz seit vielen Jahren die Unterstützung von Distriktsgesundheitssystemen (Medicus Mundi Schweiz (12), 1993). Durch diese fachliche Beratung der Mitgliedsorganisationen konnte erreicht werden, dass in der Schweiz trotz aller in den vorangegangenen Abschnitten aufgezeigten Verschiedenheiten eine verhältnismässig einheitliche Entwicklungszusammenarbeit mit einem starken Fokus auf den Gesundheitsdistrikt gefunden wurde.

Das STI und das IUED haben gemeinsam und aus eigenen Mitteln in den vergangenen Jahren mehrere Workshops organisiert, die sich mit für Distriktsgesundheitssysteme relevanten Themen beschäftigt haben (STI/IUED (13), 1991). Themen waren beispielsweise, Probleme des Dezentralisierungsprozesses, Probleme, die mit der Finanzierung von Distriktsgesundheitssystemen zusammenhängen und allgemeine Managementprobleme in Gesundheitsdistrikten. Teilnehmer dieser Workshops kamen sowohl aus Entwicklungsländern, als auch von anderen schweizerischen Nichtregierungsorganisationen, insbesondere Projektverantwortliche aus den Partnerländern und der Schweiz. Im Zusammenhang mit dem World Development Report 1993, der jedes Jahr von der Weltbank herausgegeben wird, organisierte das STI im Auftrag der Weltbank und mit einer Teilfinanzierung der DEH zwei Workshops mit internationaler Beteiligung. Die Workshops behandelten Distriktsgesundheitssysteme in Afrika und städtische Gesundheitssysteme. Wichtige Themenbereiche waren in beiden Workshops die Gemeindebeteiligung und die Entwicklung von Kriterien für die Verlaufskontrolle der Entwicklung von Gesundheitsdistrikten.

\section{Kritische Punkte bei der schweizerisches Förderung von Distriktsgesundheitssystemen}

\section{Dezentralisation und Gemeindebeteiligung}

Der periphere Ansatz und die Miteinbeziehung der Zielgruppen entspricht der schweizerischen staatlichen und nichtstaatlichen Entwicklungszusammenarbeit. Die Umsetzung dieser Grundsätze bereitet aber oft Schwierigkeiten, weil die 
Partnerstrukturen in den Projektländern wie oben aufgeführt diese Prinzipien oft nicht mit dem gleichen Enthusiasmus und der gleichen Konsequenz teilen wie die schweizerischen Entwicklungsinstitutionen und Organisationen. Hinzu kommt in manchen Ländern eine gewisse Schwerfälligkeit der staatlichen Partner. Teilweise fehlt vielleicht auch deren politischer Wille, die Entscheidungs- und Verfügungsgewalt zu dezentralisieren. Dies führt in manchen Situationen zu Verzögerungen bei der Umsetzung von Projekten. Dies trifft besonders Projekte der bilateralen Zusammenarbeit, die in die lokalen Verwaltungsstrukturen integriert sind. In einem Projekt der städtischen Gesundheitsversorgung in Tansania dauerte es beispielsweise mehr als ein Jahr bis die notwendigen Voraussetzungen und Prozeduren erfüllt waren, dass die in der Projektvereinbarung festgelegte dezentrale, d.h. von den Distriktsärzten kontrollierte Finanzmittelverwaltung von den tanzanischen Partnern tatsächlich umgesetzt werden konnte. Die Gemeindebeteiligung ist ein nicht immer sehr deutlicher Schwerpunkt der schweizerischen Projekte. Die Erklärung hierfür liegt kaum bei den schweizerischen Organisationen und Institutionen, die wie oben ausgeführt grossen Wert auf die grösstmögliche Zielgruppenbeteiligung legen. Bei den Partnerinstitutionen und Organisationen ist dies aber nicht immer der Fall. Besonders in bilateralen Projekten spielt es eine wichtige Rolle, dass die institutionellen Partner oft staatliche Strukturen sind, die, selbst wenn sie die Gemeindebeteiligung wünschen und suchen, natürlich auch ihre institutionellen Bedürfnisse haben. Schweizerische Projekte, wie bspw. das Dolakha-Ramethhap-Sindhouli-Projekt der DEH in Nepal, tragen dem Rechnung und beinhalten - sehr pragmatisch - sowohl Aspekte der Partnerstrukturunterstützung und Stärkung, daneben aber auch die direkte Arbeit mit Basisgruppen, ohne wesentliche Beteiligung der staatlichen Strukturen.

\section{Finanzierung von Gesundheitssystemen}

Die Finanzierung von Gesundheitssystemen stellt eine der grössten Herausforderungen der kommenden Jahre dar. In den Jahren nach der Unabhängigkeit versuchten viele Staaten der südlichen Hemisphäre, die Grundbedürfnisse der Bevölkerung, insbesondere die Gesundheitsversorgung, kostenfrei anzubieten. Die Erfahrung, dass Gesundheit keinen Preis hat aber Geld kostet, musste auch in diesen Ländern gemacht werden. Beispielsweise kann in vielen Gesundheitsdistriktssystemen der Staat nur sehr begrenzte Mittel für Investitionen im Gesundheitssektor zur Verfügung stellen und auch die Deckung der laufenden Kosten bereitet enorme Schwierigkeiten. Deshalb fliessen vermehrt Kostenüberlegungen in die Planung von neuen Projekten ein. Dabei ist man vom anfänglichen Optimismus, eine weitgehende Eigenfinanzierung des Gesundheitssystems zu erreichen, abgekommen und hat zu einer realistischen Perspektive gefunden, bei der es darum geht, wenigstens einen Teil der Kosten durch die Erhebung von Nutzungsgebühren oder durch angepasste Versicherungssysteme lokal zu decken. Mittelfristiges Ziel ist es, damit den Subventionsbedarf der öffentlichen Hand zu reduzieren. Konkrete, positive Erfahrungen wurden in grösserem Rahmen in Projekten in Westafrika (Benin und Tschad) gemacht. Ein wesentlicher Aspekt bei der 
Finanzierung von Gesundheitssystemen ist die Notwendigkeit, die eng mit der oben ausgeführten Dezentralisierung zusammenhängt, lokale "Geld-Zyklen" einzuführen, d.h. dass Gesundheitsdienste ihre Einnahmen lokal verwalten und gegenüber der Bevölkerung verantworten. Die Verwendung der Mittel muss transparent sein und von der Bevölkerung nachvollzogen werden können. Der letzte Aspekt ist für die Akzeptanz bei der Bevölkerung und damit die Nachhaltigkeit von wesentlicher Bedeutung.

Der Subventionsbedarf von Distriktsgesundheitssystemen wird sich mittelfristig kaum ändern, und Überlegungen sind im Gange, inwiefern nicht auch externe Geber einen Teil der Funktionskosten übernehmen sollten, um minimale Gesundheitsdienstleistungen sowohl im präventiven als auch im kurativen Bereich sicherzustellen. Die Begründung für derart grundlegende Änderungen in der Entwicklungszusammenarbeit, die bisher aus Gründen der Nachhaltigkeit von der Übernahme von laufenden Kosten zurückgeschreckt ist, ist die sich immer mehr durchsetzende Erkenntnis (World Bank (14), 1993), dass es sich entwicklungspolitisch "rechnet" in die Gesundheit der Zielbevölkerung in den Partnerländern zu investieren.

\section{Qualitätsaspekte}

Unabdingbar verknüpft mit der Finanzierung von Gesundheitsdistriktssystemen ist die Verbesserung der Qualität der Dienstleistungen. Wichtige Aspekte sind einerseits auf der individuellen Ebene, dass die Bevölkerung bereit ist, nur für gute Qualität zu bezahlen. Beim Versuch über die Qualität der Gesundheitsdienstleistungen muss die empfundene, von der Bevölkerung erfahrene Qualität von der rein technisch definierten Qualität abgegrenzt werden. Obwohl Erfahrungen in Tansania gezeigt haben, dass die technische Qualität der Dienstleistungen im öffentlichen Gesundheitssektor besser ist als ihr Ruf, besteht ein enormer Handlungsbedarf, um die Qualität der Dienstleistungen anzuheben. Eine gute Qualität, sowohl im technischen als auch im von der Bevölkerung empfundenen Bereich, ist letztendlich eine Grundvoraussetzung dafür, dass ein Gesundheitssystem seinem Anspruch, d.h. die Gesundheit zu erhalten, bzw. Krankheit vorzubeugengerecht werden kann. Volkswirtschaftlich gesehen ist die Verbesserung der Qualität der Gesundheitsdienstleistungen deshalb wichtig, da nur eine gute technische und empfundene Qualität der Leistungen eines Gesundheitssystems die enormen Ausgaben für diesen Bereich rechtfertigt, insbesondere wenn in allen Ländern der Norden und die Schweiz müssen hier voll eingeschlossen werden - nur begrenzte Ressourcen für den Gesundheitsbereich zur Verfügung stehen, und eine optimale Nutzung der verfügbaren Mittel deshalb unbedingt notwendig ist.

\section{Unterstützung des Distriktkonzepts in internationalen/multilateralen Institutionen}

Das Distriktskonzept hat in multilateralen Organisationen, wie die Weltgesundheitsorganisation (WHO) oder das United Nations Development Program (UNDP), 
noch nicht die notwendige Akzeptanz. Nichtregierungsorganisationen, wie beispielsweise Medicus Mundi International, haben die Bedeutung des Konzepts schon länger erkannt, bzw. teilweise aktiv zur Ausarbeitung des Distriktskonzepts beigetragen. In der Schweiz gibt es eine Reihe von Organisationen und Institutionen, die eine umfangreiche Erfahrung in der Unterstützung von Distriktsgesundheitssystemen besitzen. Auf diesem Hintergrund wäre es wünschenswert, auch in internationalen Gremien, in denen die Schweiz vertreten ist, das Konzept konsequenter zu unterstützen und gezielt zu fördern. Dies würde eine logische und konsequente Ergänzung zu den distriktsorientierten Interventionen des schweizerischen Entwicklungszusammenarbeit darstellen.

\section{Lösungsansätze:}

\section{Partnerschaftlicher Ansatz auf allen Ebenen, Beteiligung der Zielgruppen, Miteinbezug der Betroffenen}

Der partnerschaftliche Ansatz ist nicht nur für Distriktsgesundheitssysteme ein Paradigma der Entwicklungszusammenarbeit. Nur hierdurch können dauerhafte und nachhaltige Lösungen erreicht werden. Formal arbeiten fast alle schweizerischen Organisationen und Institutionen mit lokalen Partnern zusammen. Der partnerschaftliche Ansatz beinhaltet eine Diskussion und die Bereitschaft zum Kompromiss. Die grundsätzliche Bereitschaft ist bei schweizerischen Organisationen und Institutionen in hohem Masse vorhanden. In der Praxis kommt es aber immer wieder zu Schwierigkeiten, in dem die Grundregeln wie "zuhören" und "aufmerksam sein für die Erwartungen der Partner und deren Initiativen" vergessen werden.

\section{Audit von Aktivitäten, Monitoring und Evaluierung}

Die Grenzen von Audit zur operationellen Forschung sind fliessend. In den vergangenen Jahren wurden bspw. in den Projekten des STI und IUED grosse Anstrengungen unternommen, um die kontinuierliche Überwachung und Kontrolle von Projektimplementierungen zu verbessern. Wichtig bei der kontinuierlichen Verlaufskontrolle waren nicht nur die Bestimmung von Ergebnissen, sondern v.a. auch die Beobachtung von Prozessen. Die Kenntniss der Prozesse, die zu Ergebnissen geführt haben, ist wichtig, um Faktoren zu erkennen, die für die Übertragbarkeit von Konzepten und Ansätzen von Bedeutung sind. Durch die Kostenanalyse von Gesundheitsprojekten im Tschad wurden wichtige Grundlagen geschaffen, die in Zukunft die Budgetierung von neuen Projekten erleichtern werden. Vor allem kann durch diese Analyse den Partnerländern aufgezeigt werden, welche laufenden Kosten langfristig für ein funktionierendes Distriktgesundheitssystem anzusetzen sind. Ein anderes Schwerpunktgebiet der Entwicklungszusammenarbeit in diesem Bereich ist die Entwicklung der menschlichen Ressourcen (s. entsprechendes Kapitel). Da für diesen und andere Bereiche 
sehr wenig gesichertes Wissen für die südliche Hemisphäre vorliegt, kommt der Gesundheitssystemforschung eine grosse Bedeutung zu.

\section{Gesundheitssystemforschung}

Die operationelle Forschung hat traditionell einen hohen Stellenwert in der schweizerischen Unterstützung von Distriktsgesundheitssystemen. Sie wird bedauerlicherweise in der Schweiz manchmal als "akademische" Forschung missverstanden, die mit den prioritären Problemen der Partnerländer nur bedingt - wenn überhaupt - etwas zu tun hätte.

Die Kritiker dieser angewandten Forschung übersehen, dass die Inhalte durch lokale Gegebenheiten und Problemen bestimmt werden. Die Forschungsthemen umfassen deshalb ein weites, praxisnahes Spektrum. Wichtig wird in den kommenden Jahren die Entwicklung und Validierung von distriktsbezogenen Ansätzen zur Problemidentifikation und deren Lösungen sein. Eine besondere Situation ergibt sich in der Schweiz dadurch, dass das STI und das IUED einerseits akademische Einrichtungen sind und andererseits direkte Projektdurchführungsverantwortung besitzen. Diese in Europa sehr seltene Verbindung ermöglicht es, dass einerseits der Praxisbezug der operationellen Forschung sichergestellt ist, und andererseits Erfahrungen für andere Projektdurchführungen gewonnen werden können. Die Tatsache, das sich operationelle Forschung aber nicht nur im universitären Umfeld findet, unterstreicht, dass das Ziel der Gesundheitssystemforschung, dh. die Entwicklung von angepassten Lösungen, von vielen Schweizer Organisationen und Institutionen gut verstanden wird.

Um derartige Forschung auch in Zukunft betreiben zu können ist es notwendig, sowohl im Süden als auch in der Schweiz die vorhandene Forschungskapazität nicht nur zu erhalten sondern auch weiter auszubauen. Der Initiative der DEH und der Schweizerischen Akademie für Naturwissenschaften (DEH/SANW (15), 1993) zur Förderung der Forschung in Entwicklungsländern kommt hier eine grosse Bedeutung zu, und es bleibt nur zu hoffen, dass die notwendigen Mittel zur Verfügung stehen werden.

\section{Managementsupport}

Der allgemeine Trend der distriktsorientierten Unterstützung bewegt sich von der rein kurativmedizinischen Seite zur Unterstützung der Gesundheitsadministration. Dies spiegelt sich beispielsweise darin wieder, dass Planung und Evaluierung einen grossen Stellenwert besitzen und damit fast zwangsläufig rein medizinische Ziele, wie beispielsweise die Reduktion der Kindersterblichkeit, in den Hintergrund treten und operationelle Ziele, wie beispielsweise die Einrichtung eines angepassten Gesundheitssystems unter Einbeziehung der Bevölkerung, in den Vordergrund treten. Das ändert natürlich nicht das Oberziel der schweizerischen Entwicklungszusammenarbeit im Gesundheitssektor, das darin besteht, den Gesundheitszustand der Zielbevölkerung in den Partnerländern der südlichen Hemisphäre zu verbessern. 


\section{Koordination und Versuch, Synergien zwischen schweizerischen Projekten zu fördern}

Trotz einer gewissen Koordination der schweizerischen Aktivitäten in der Unterstützung distriktsorientierter Gesundheitsprojekte durch Medicus Mundi beschränkt sich diese fast ausschliesslich auf den Gesundheitssektor. Auf Grund der geographischen Verteilung der Projekte kommt es nur sehr begrenzt zu Synergien. Ein positives Beispiel ist die François-Bauer-Stiftung, die in Ifakara/Tansania den Versuch unternimmt, komplementär zu ebenfalls von schweizerischen Mitteln unterstützten Aktivitäten am St Francis Designated District Hospital und an dem oben erwähnten Ifakara Centre mit seiner Distriktunterstützungskomponente die Medikamentenversorgung durch die Schaffung einer nicht profitorientierten Apotheke zu unterstützen. In Ansätzen besteht eine Arbeitsgruppe von verschiedenen schweizerischen Organisationen, die in Tansania intervenieren. Im Augenblick beschränkt sich diese Gruppe auf gegenseitige Information, aber weitergehende Kooperationen sind denkbar und möglich. Ohne wesentliche Beteiligung des Gesundheitssektors gibt es in der Schweiz derartige Koordinationsgruppen auch für verschiedene Länder in Lateinamerika. Ein abschliessendes Beispiel eines im Ansatz guten Versuchs, die schweizerische Entwicklungszusammenarbeit zu koordinieren, ist das Länderprogramm der DEH für Tansania, in dem einerseits Gesundheits- und Infrastrukturmassnahmen als wesentliche Komponenten enthalten sind, und der Ansatz der Gemeindebeteiligung das Bindeglied und die Grundlage der Zusammenarbeit darstellt.

Die Koordination und die Stärkung der Synergien kann wesentlich dazu beitragen, dass in partnerschaftlichen Verhältnissen die Qualität von Gesundheitsdienstleistungen in der südlichen Hemisphäre mit Schweizer Unterstützung verbessert und die Nachhaltigkeit von Distriktsgesundheitssystemen gesichert wird.

\section{Anmerkungen / Literatur}

1. Tarimo E.: Towards a healthy district Organizing and managing district health systems based on primary health care. World Health Organization, Geneva, 4 (1991).

2. World Health Organization: The Challenge of Implementation District Health Systems for Primary Health Care. World Health Organization; Geneva (1988).

3. Medicus Mundi International: District Health System and the Health Care Revolution. Conference Report International Colloqium. Medicus Mundi International, Brussels (1989).

4. Fischer F.H.: Der Missionsarzt Rudolf Fisch und die Anfänge medizinischer Arbeit der Basler Mission and der Goldküste (Ghana). Murken-Altrogge, Herzogenrath (1991).

5. Cadotsch A.F.: Entwicklungszusammenarbeit im Aufbau eines Basisgesundheitsdienstes. Acta Tropica 23, 189-203 (1977).

6. Institut of Development Studies, Swiss Academy of Science, Swiss Development Cooporation: Scientific Cooperation with developing countries. Who does what in Switzerland. A preliminary survey of institutions, research projects, researchers. IUED, Geneva; ASSN, Bern; DEH, Bern (1992). 
7. Tanner M., Lengeler Ch., Lorenz N.: From the efficacy of disease control tools to community effectiveness. Case studies from the biomedical and health systems research activities of the Swiss Tropical Institute in Africa. Transactions of the Royal Society of Tropical Medicine and Hygiene; Vol. 87 (1993): 518-523.

8. Lengeler $\mathrm{CH}$., Sala-Diakanda D., Tanner M.: The distribution of simple questionnaires through an existing administrative system: new approach to health interview surveys. Health Policy and Planning. 7, 10-21 (1992).

9. Teuscher T., Armstrong Schellenberg J.R.M., de Azevedo B., Hurt N.,Smith T., Hayes R., Masanja H., Silva Y., Lopez M.C., Kitua A., Kilama W., Tanner M., Alonso P.L.: SPf66, a chemically synthesised subunit malaria vaccine, is safe and immunogenic in Tanzanians exposed to intense malaria transmission. Vaccine (in press) (1994).

10. Izurieta H., Do Rego D.: Enquête Epidémiologique sur l'Etat Nutritionnel dans la SousPréfecture de Zogbodomey; IUED, Genève (1990).

11. Swiss Tropical Institute: Research Report 1991-1993. Schweizerisches Tropeninstitut, Basel (1993).

12. Medicus Mundi Schweiz: Standortbestimmung und aktuelle Fragen Berichte aus unseren Mitgliederorganisationen. Medicus Mundi Schweiz, Basel (1993).

13. Schweizerisches Tropeninstitut, Institut Universitaire d'Etudes du Développement: Joint Workshop STIIIUED on Health District Management. Schweizer Tropeninstitut, Basel (1991).

14. World Bank: World Development Report 1993. World Bank, Oxford University Press, Washington (1993).

15. DEH, Schweizerische Akademie für Naturwissenschaften: Schweizerische Strategie zur Förderung der Forschung in Entwicklungsländern. Bern (1993). 